\title{
Un par de pliegos enrevesados de Miguel Barnades Mainader y Esteban de Prado en el Herbario del Real Colegio Alfonso XII de San Lorenzo de El Escorial (Madrid)
}

\author{
Antonio González-Bueno ${ }^{1}$, María A. Carrasco ${ }^{2}$ y Domingo Perea ${ }^{3}$
}

\begin{abstract}
Resumen: González-Bueno, A.; Carrasco, M. A. \& Perea, D. 2015. Un par de pliegos enrevesados de Miguel Barnades Mainader y Esteban de Prado en el Herbario del Real Colegio Alfonso XII de San Lorenzo de El Escorial (Madrid). Bot. Complut. 39: 115-119.

Se presentan dos pliegos de Umbelliferae procedentes del Herbario Barnades que se conservan en el Herbario del Real Colegio Alfonso XII de San Lorenzo de El Escorial, herbario que denominamos como RCAXII. Uno de ellos, Selinum carvifolia, fue recolectado en la Sierra de Gredos por Miguel Barnades Mainader e identificado por su hijo Miguel Barnades Clarís. El otro, Tragium flavellifolium, lo herborizó en Mieres (Asturias) Esteban de Prado y fue identificado por Mariano La Gasca.
\end{abstract}

Palabras clave: colecciones históricas, herbario Barnades.

Abstract: González-Bueno, A.; Carrasco, M. A. \& Perea, D. 2015. A couple of embroiled sheets of Miguel Barnades Manaider and Esteban de Prado in the Herbaria of Real Colegio Alfonso XII of San Lorenzo de El Escorial (Madrid). Bot. Complut. 39: 115-119.

In this paper we present a couple of sheets of Umbelliferae that are preserved in the RCAXII herbaria. One of them, Selinum carvifolia, where collected in the Gredos Mountains by Miguel Barnades Mainader and was identified by his son Miguel Barnades Clarís. The other, Tragium flabellifolium, was collected in Mieres (Asturias) by Esteban de Prado and identified by Mariano La Gasca.

Key words: historical collections, Barnades herbarium.

\section{INTRODUCCION}

Entre las colecciones conservadas en el Herbario del Real Colegio Alfonso XII de San Lorenzo de El Escorial (RCAXII), cuya historia explicamos en Carrasco et al. (1996) y cuyas colecciones fueron relacionadas en Carrasco et al. (2001), se guardan un par de pliegos, de pequeño tamaño, con anotaciones en el reverso, cuya estructura y contenido se corresponden con las recolecciones realizadas por Miguel Barnades y Mainader (Ibáñez et al. 2006). El RCAXII viene así a sumarse a los herbarios BC, BM, C, LINN, MA (Stafleu \& Mennega 1992: 329) actuales depositarios de los pliegos compilados por este botánico. Este herbario no está registrado en Thiers, B. pero lo venimos denominando RCAXII desde que dimos cuenta de su aparición (Carrasco et al. 1996).

Miguel Barnades y Mainader (Puigcerdá, Barcelona, 1708 / Madrid, 1771) fue médico de Carlos III (1716-1788) y del XII duque de Alba, Fernando de Silva y Álvarez de Toledo (1714-1776); formado en Montpellier, bajo la dirección de François Boissier de Sauvages de Lacroix (1706-1767), ejerció como 'primer profesor' del Real Jardín Botánico entre 1764 y 1771, tras la muerte de José Quer Martínez (1695-1764), quien le antecedió en el cargo. Publicó unos Principios de botánica... (Barnades 1767), en los que intentó adaptar al castellano el léxico descriptivo de la botánica (Camarasa 1989: 59; Nomdedeu Rull 2012); dejó inédito un Specimen Florae hispanicae..., con la descripción de unos dos mil taxones, ilustrados con un centenar de dibujos, e información sobre los vulgarismos, usos y localidades de las plantas (Colmeiro 1858: 166).

En vida, Miguel Barnades y Mainader fue generoso con los pliegos de su herbario, no dudo en ofrecérselos a quien por ellos se interesaran, es el caso de Ignacio Jordán de Asso y del Río (1742-1814), quien, a su vez, no

\footnotetext{
${ }^{1}$ Departamento de Farmacia y Tecnología Farmacéutica, Universidad Complutense de Madrid, agbueno@farm.ucm.es

${ }^{2}$ General Pardiñas, 118, 28006 Madrid, ma.carrascosalazar@gmail.com

${ }^{3}$ Real Colegio Alfonso XII, 28200 San Lorenzo de El Escorial, Madrid, domingo@colalfonsoxii.com

Recibido: 16 junio 2014. Aceptado: 4 agosto 2014.
} 
menos desprendido, no dudo en regalárselos a Antonio José Cavanilles y Palop (1745-1804), cuando éste se los solicitó como material de estudio (González Bueno 2002: 113). Tras su muerte, las colecciones de Miguel Barnades y Mainader pasaron a su hijo, Miguel Barnades y Clarís (1750-1801); éste cedió sus manuscritos, al menos el Specimen Florae hispanicae..., que él mismo se había ocupado de continuar, a Pedro Felipe de Valencia y Codallos (1767-ca. 1815), segundo conde de Casa Valencia; de manos de su viuda, María Antonia de Junco y Rosales, pasaron, en 1817, a las de Mariano La Gasca y Segura (1776-1839) quien continuó adicionando comentarios; al menos así se hace constar en el catálogo de la subasta que el librero Vicente Salvá Pérez (1786-1849) organizó en Londres en 1829, donde el manuscrito de los Miguel Barnades acompaña a un Herbarium pictum hispanicum..., compuesto por seis tomos, con 1383 dibujos de plantas (Salvá 1826-1829, 2: 107; Colmeiro 1858: 73); la obra debió quedar en poder de La Gasca y, junto al resto de su biblioteca, quedó depositada en el Instituto de Bachillerato de Málaga (Pérez Rubín 2010), de donde, en 1863, Mariano de la Paz Graells y de la Aguera (1809-1898), director del Museo de Ciencias y del Jardín de Madrid, adquirió el Herbarium pictum hispanicum..., tras la intermediación de Miguel Colmeiro y Penido (1816-1901); la parte descriptiva, que se conservó, en Málaga, junto al resto de la biblioteca lagascana, no llegó al Real Jardín.

El herbario de los Barnades siguió un camino diferente; Miguel Colmeiro (1858: 166) señala que «Del herbario de los Barnades solamente restan algunas plantas, que pasaron á poder de Pavón, y del de este al de otros botánicos y al de la Academia de Ciencias Naturales de Barcelona.» José Antonio Pavón y Jiménez-Villanueva (1754-1840) distribuyó los pliegos barnesianos, junto al resto de los que fue poseedor, entre los botánicos europeos dispuestos a comprarlos: Jules Paul Benjamin Delessert (1773-1847), Philip Barker Webb (1793-1854) y Pierre Edmond Boissier (1810-1885), entre ellos, lo que propició la dispersión de las colecciones por un buen número de herbarios europeos (Rodríguez Nozal 1994); el grueso de la colección, 817 pliegos, fue adquirido -junto al resto del herbario Pavón-, en la primavera de 1838 , por la Real Academia de Ciencias y Artes de Barcelona, actuando Mariano de la Paz Graells como mediador. La Real Academia los cedió, en 1925, al Museo de Ciencias Naturales de Barcelona, cuya sección de Botánica es el origen del Instituto Botánico de Barcelona, donde actualmente se conservan (Ibáñez 2006: 97-114; Ibáñez et al. 2009).

\section{RESULTADOS}

Para la transcripción de las etiquetas se sigue el protocolo propuesto por Burdet et al. (1979: 66).

\section{Selinum carvifolia (L.) L., RCAXII 1743}

Selinum Carvifolia Linn. // anpotius palustre Crann umbell.? // ¿Selinu fol. triplicato pinnatis pinnulis latiuscu? / trifidis et simplicitis. Hall. stirp. emend. p. $356 / \mathrm{n}$. 802 ic. 20 Gm. t. 48 tom 2. // Oeder tab. 257 inflor. Dan. nra bene expr. // Sel. palustre Linn. // August. 1764. // Loc. herbosa ad calc. Rup. el Risco de los Morzones // prope Lacunam de Gredos. (Fig. 1 y 2).

Observaciones.- Uno de los ejemplares, completo, con hojas inflorescencia e infrutescencia, está montado sobre un folio que lleva en el reverso la información transcrita. Está manuscrito en tinta. Otro ejemplar con inflorescencias e infrutescencias está montado en pliego aparte.

El pliego se incluye en una amplia serie de herborizaciones en tierras abulenses, realizadas por Miguel Barnades y Mainader (Miguel Barnades, padre), de las que nos queda constancia entre el septiembre de 1759 y el septiembre de 1772 y que podríamos vincular a las estancias ve-

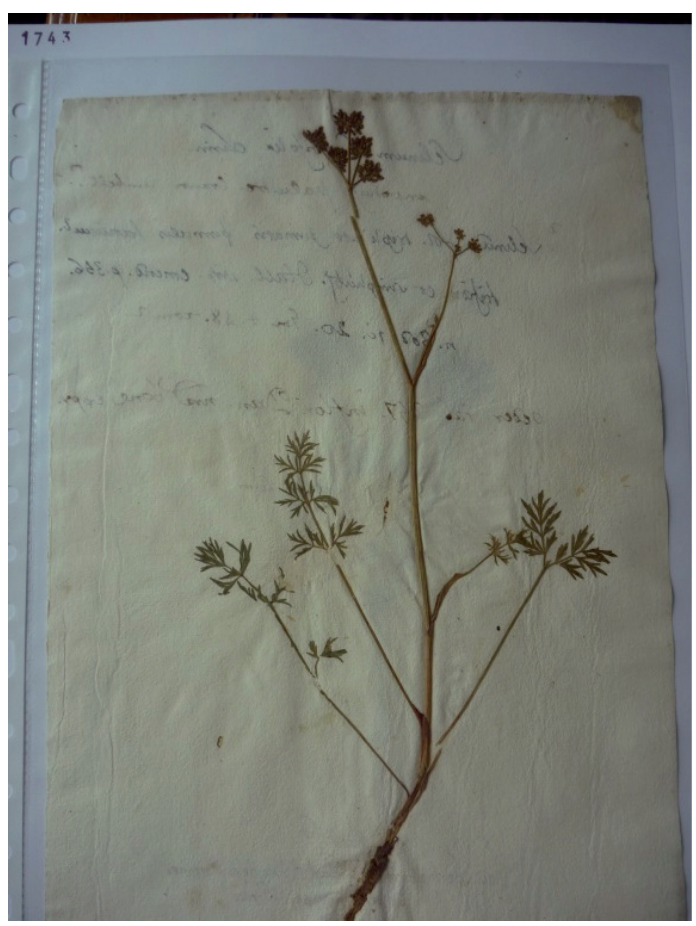

Fig. 1- Selinum carvifolia, anverso del pliego RCAXII 1743. 


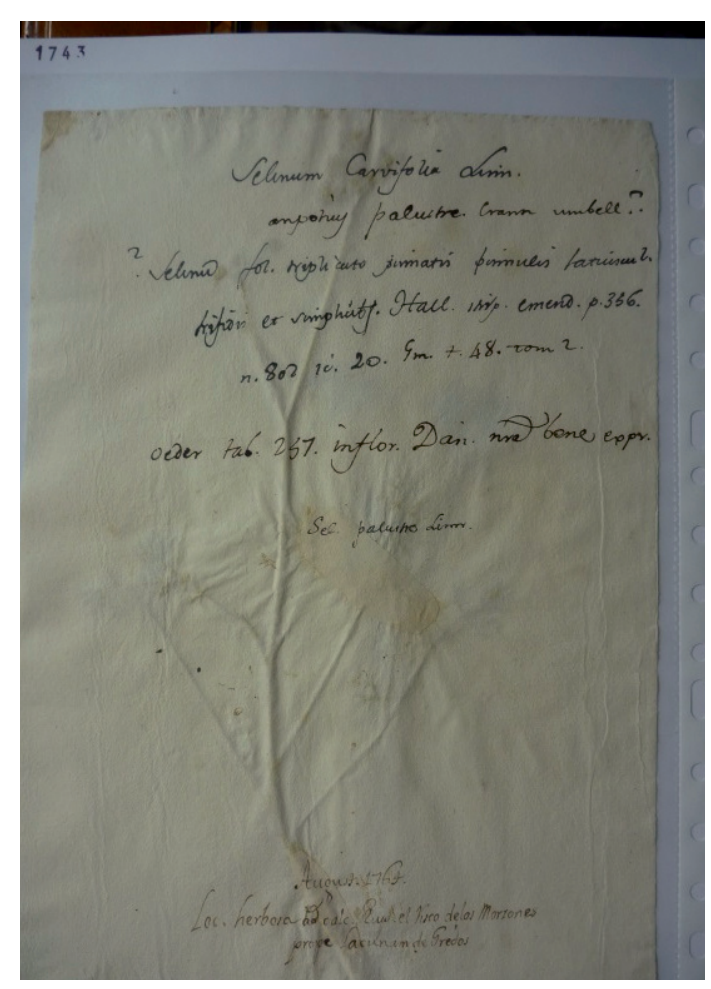

Fig. 2- Selinum carvifolia, reverso del pliego RCAXII 1743.

raniegas de Fernando de Silva y Alvarez de Toledo en el Palacio de Piedrahita, levantado entre 1755 y 1766, por el arquitecto francés Jacques Marquet (1710-1782), por expreso deseo de éste XII duque de Alba, al que Miguel Barnades y Mainader acompañaría en el cargo de médico de su Casa. La mano que escribió las líneas infrapaginales: «August. 1764. // Loc. herbosa ad calc. Rup. el Risco de los Morzones // prope Lacunam de Gredos» se corresponde con la de Miguel Barnades padre.

Las anotaciones sinonímicas que acompañan al binomen linneano - 'Selinum carvifolia' L., Sp. Pl. (ed. 2): 350. 1762- aluden a: 'Selinum palustre' Crantz, Cl. Umbell. Emend.: 60. 1767; 'Selinum [802]' Haller, Hist. Stirp. Helv.: 356. 1768; 'Selinum [18]' Gmelin, Fl. Sibir. 1: 204. 1747; 'Selinum [257]' Oeder, Fl. Dan.: 5, t. 257. 1766; textos que, por su fecha de edición, bien pudieron ser consultados por Miguel Barnades y Mainader, fallecido en 1771; sin embargo, la letra se asemeja más a la de su hijo, Miguel Barnades y Clarís, a quien también cabría responsabilizar, por la similitud gráfica, de la anotación «Sel. palustre L.», Sp. Pl.: 244. 1753, aun cuando ésta podría ser de momento distinta al resto de las anotaciones a él atribuidas. Además de la similitud de la letra indicativa de la localidad con la de otras anotaciones manuscritas atribui- das a Miguel Barnades y Mainader (Ibáñez et al. 2009: 37), no parece posible que el material fuera recolectado por un imberbe Miguel Barnades y Clarís, con apenas trece años de edad.

Entre los pliegos conservados en $\mathrm{BC}$, pertenecientes a la colección Barnades, se guarda uno (BCBernades-291), etiquetado como «Selinum carvifolia [m.i.] / Ex Prat. primo occur. ad lovam via ab Opp. Miraflores ad Convallem / de Bustar Viejo» [m. i]», al que acompaña una etiqueta de revisión, debida a Samuel Pyke (24/1/2006), quien le señala como Peucedanum carvifolia Crantz. ex Vill.; es posible que, en algún momento, el pliego conservado en RCAXII fuera considerado como 'duplicado' del que ahora se custodia en BC.

El segundo pliego de nuestro interés tiene las mismas dimensiones y conserva la misma estructura en la disposición de las anotaciones que el atribuido a Miguel Barnades y Mainader, pero su grafía es bien diferente.

\section{Tragium flavellifolium Lag., RCAXII 1742}

Anverso: Tragium flavellifolium Lag. (m. La Gasca) Reverso: Differt â Tragio Colum. rad. setis non coro-

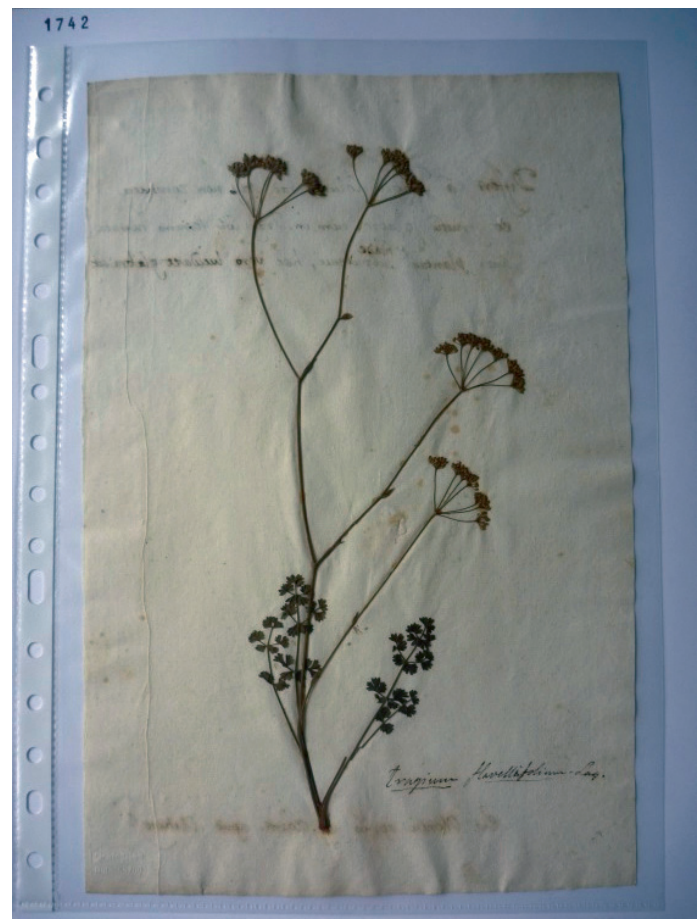

Fig. 3- Tragium flabellifolium, anverso del pliego RCAXII 1742 . 


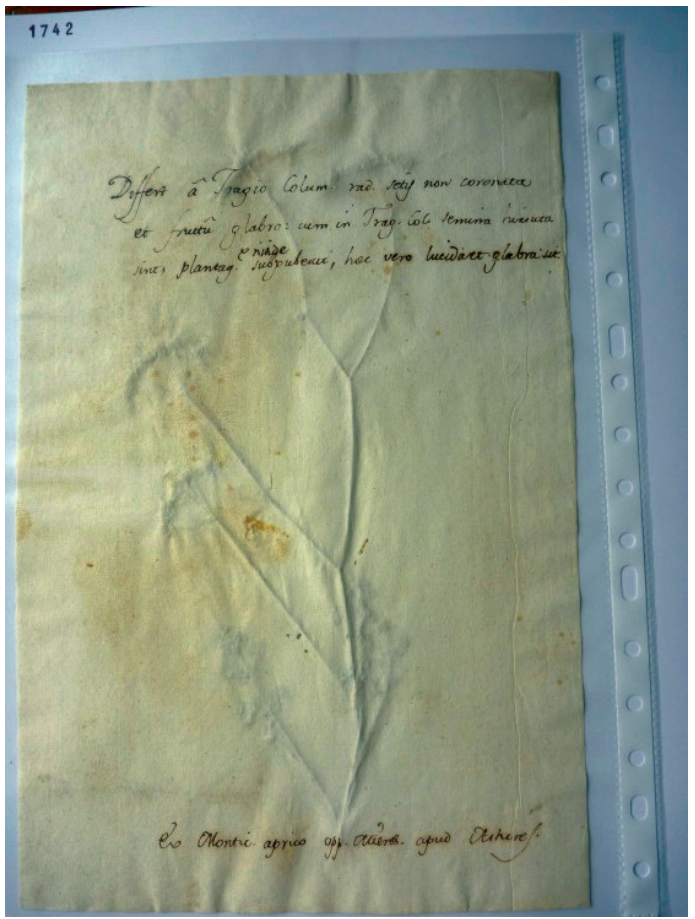

Fig. 4- Tragium flabellifolium, reverso del pliego RCAXII 1742.

nata / et fructû glabro: cum in Trag. Col. semina hirsuta / sint, plantag, rigide subpubescet, hoc vero lucidaret glabra s. it. // Ex Monti aprico opp. Mieres. apud Astures.

Observaciones. - Se trata de un pie de planta completo, con hojas, inflorescencias y frutos. Está pegado sobre un folio que tiene escrita la información de la etiqueta. En el anverso: «Tragium flavellifolium» (m. La Gasca).

La letra indicando la localidad, cuya ubicación infrapaginal recuerda al resto de la colección Barnades, se debe a Esteban de Prado (m. 1783) (Archivo RJB, signatura I,21,1,7), corresponsal del Real Jardín Botánico en tierras de Mieres, al menos desde 1767 (Laínz 1985, Añón 1987: 35, Carlón 2006); quizás deban atribuirse también a su trabajo los otros pliegos que, bajo la indicación de mano desconocida o de copista, se conservan en la colección Barnades del Herbario BC procedentes de Mieres y su vecindad: Puerto del Áramo y San Tirso; incluso es probable que se deban a él los que, con letra de Miguel Barnades y Mainader, indican topónimos mierenses y ovetenses, fechados durante el mes de mayo de 1762, conservados en $\mathrm{BC}$, habida cuenta que por estas mismas fechas nos constan recolecciones de Barnades padre en la zona abulense de Villafranca de la Sierra y Piedrahita (Ibáñez 2006: 97-114, Ibáñez et al. 2009)
La letra de las observaciones taxonómicas y la propia determinación del ejemplar corresponden a Mariano La Gasca y Segura (1776-1839). Es conocido el viaje botánico por tierras leonesas y asturianas que realizara este botánico, aún bajo la dirección de Cavanilles, en el verano de 1803, pero parece que no pisó Mieres ni sus proximidades (Pérez de Castro 1971, Laínz 1978), por lo que no puede atribuírsele esta recolección.

El nombre propuesto por Mariano La Gasca, 'Tragium flavellifolium', no llegó a ser válidamente publicado; la descripción del género Tragium Spreng. fue realizada por Curt [Kurt] Polycarp Joachim Sprengel (1766-1833) en Plantarum umbelliferarum Denuo disponendarum prodromus... (Halae: typis Hendelianis, 1813) para agrupar en él algunos taxones de una sección de Pimpinella L.; Tragium columnae Spreng. resulta ser un nuevo nombre construido para el antiguo Pimpinella columnae Vill.

Mariano La Gasca hizo públicas sus opiniones sobre el género Tragium Spreng. dentro de su «Dispositio umbelliferarum...» incluida en el segundo número de sus Amenidades naturales de las Españas, ó bien disertaciones varias Sobre Las Producciones Naturales Espontáneas, ó Conaturalizadas en los Dominios Españoles... (Madrid: Por Ibarra, Impresor de cámara de S. M., 1821); allí, en la página 104 reconoce:

«64. Tragium. ( Spreng.) Polachena ovata, vel ovato-conica, dense pubescens, jugis tenuissimis; valleculis convexiusculis, latusculis paralello-convergentibus. Petala pseudo-emarginata. Involucr. universale \& partiale. 0. vel submonophylium. $=$ Herbae glaucescentes vel cincrascentes. Umbellae composita, nubiles nutantes. Flores albi, petalis erectiusculis, extus pubescentibus. Commissura planiuscula vel excavata. Folia radicalia 1-2-3 pinnatisecta. Foliolis sape rotundatis, cuneatisve.

Sp. cert. 1. Tr. Columna: Spr. 2. peregrinum. Spreng. 3. villosum. Spreng.»

De forma que este 'Tragium flavellifolium' no estaba para él aún, en 1821, entre las 'especies ciertas' de las pertenecientes a este género que crecen en nuestro suelo. Salvo por la presencia de este pliego, a todas luces identificado con posterioridad a 1821, no tenemos constancia de que Mariano La Gasca volviera a escribir sobre la taxonomía de este género (Gamarra 1993).

Quizás este pliego guarde relación con el citado bajo el nombre Pimpinella tragium Vahl (Willkomm \& Lange 1880: 97-98), donde consta como localidad «in rupibus, campis siccis, solo praecipue calcáreo, rarius: Astur.: Mie- 
res (Barnad. In hb VAHL!), pliego que no ha sido localizado entre los materiales de Martin Vahl (1749-1804) con- servados en el Museo Botánico de Copenhague (Laínz 1963: 80).

\section{BIBLIOGRAFÍA}

AÑón FeLíu, C. 1987. Real Jardín Botánico de Madrid: sus orígenes, 1755-1781. Real Jardín Botánico (CSIC), Madrid.

Barnades y Mainader, M. 1767. Principios de Botánica, sacados de los mejores escritores, y puestos en lengua castellana... Parte primera con las estampas necesarias. En la Imprenta de Antonio Pérez de Soto, Madrid.

Burdet, H. M.; Charpin, A. \& JaCQuemoud, F. 1979. Types nomenclaturaux des taxons décrits par Boissier, Leresche et Levier à la suite de leurs excursions en Espagne de 1878 et 1879. Mém. Soc. Bot. Genève 1: 63:82.

Camarasa, J. M. 1989. Botànica i botànics dels Països Catalans. Enciclopèdia Catalana. Barcelona.

CARLÓN, L. 2006. Dos siglos de botánica científica en Gijón (1803-2006). Carbayeda 3: 16-20.

Colmeiro Penido, M. 1858. La botánica y los botánicos de la Peninsula Lusitana. Estudios bibliográfcos y biográficos. Imprenta y estereotipia de M. Rivadeneyra, Madrid.

Carrasco, M. A.; Martín-Blanco, C. J.; García, A. \& Perea, D. 1996. Plantas de D. Estanislao Vayreda en el Real Colegio Alfonso XII de San Lorenzo de El scorial, Madrid, Anales Jard. Bot Madrid 54 (1): 589-594.

Carrasco, M. A.; Martín-Blanco, C. J. \& Perea, D. 2001. Herbaria of E. Carreño (1818-1841) and of M. P. Graells (18091898) discovered in the Real Colegio Alfonso XII of San Lorenzo de El Escorial, Madrid, Spain. Taxon 50: 587-591.

GamarRA GamarRA, R. 1993. Nomina plantarum in bibliographia lagascana provenientia. Fontqueria 36: 1-58.

GonzÁlez Bueno, A. 2002. Antonio José Cavanilles (1745-1804). La pasión por la Ciencia. Fundación Jorge Juan, Madrid.

IBAÑEZ CoRTINA, N. 2006. Estudis sobre cinc herbaris històrics de l'Institut Botànic de Barcelona. [Tesis doctoral, dirigida por J. M. Montserrat e I. Soriano]. Universitat de Barcelona, Departament de Biologia Vegetal, Barcelona.

Ibáñez, N.; I. Soriano \& J. M. MontSerrat. 2009. L'herbari Bernades a l'Institut Botànic de Barcelona (BC). Collect. Bot. (Barcelona) 28: 31-63.
LA GASCA, M. 1821. Dispositio umbelliferarum. Amenidades naturales de las Españas 1(2): 87-111.

Laínz, M. 1963. Sobre las recolecciones botánicas mierenses del siglo XVIII. Bol. Inst. Est. Asturianos. Supl. Ciencias 8: $78-83$

LaInz, M. 1978. Mariano de Lagasca en la Cordillera (julioagosto de 1803). Torrecerredo, $2^{a}$ época, 3: 26-29.

Laínz, M. 1985. Los albores de la botánica en Asturias. Colegio de la Inmaculada, Gijón.

Nomdedeu Rull, A. 2012. Los Principios de botánica (1767) de Miguel Barnades i Mainader y la creación de léxico botánico en español. Quaderns de filología. Estudis lingüístics 17 [Lengua y ciencia. Recepción del discurso cientifico]: 225-242.

PéreZ De CASTRO, J. L. 1971. Del viaje de Don Mariano La Gasca por Asturias. Bol. Inst. Est. Asturianos 72: 61-91.

Pérez-RuBín, J. 2010. Los materiales botánicos de Mariano La Gasca llegados a Málaga en 1834. Acta Bot. Malacitana 35: 215-219.

RodRÍGUEZ NoZAL, R. 1994. Las colecciones americanas generadas por las expediciones botánicas de la España Ilustrada: un análisis de su dispersión. Llull 17: 403-436.

Salvá, V. 1826-1829. A catalogue of Spanish and Portuguese books, with occasional library and bibliographical remarks. M. Calero, London.

Stafleu, F. A. \& Mennega, E. A. 1992. Taxonomic literature. A selective guide to botanical publications and collections with dates, commentaries and types, Supplement I: A-Ba. Koeltz Scientific Books, Köningstein.

THIERs, B. [continuously updated]. Index Herbariorum: A global directory of public herbaria and associated staff. New York Botanical Garden's Virtual Herbarium. http://sweetgum.nybg.org/ih/ [consultado en agosto de 2014].

Willkomm, M. \& Lange, J. 1880. Prodromus Florae Hispanicae, 3. E. Schweizerbart, Stuttgart. 